\title{
Technology Choices of Undergraduate Engineering Students for Solving Cal- culus Questions
}

\section{Dr. Emre Tokgoz, Quinnipiac University}

Emre Tokgoz is currently an Assistant Professor of Industrial Engineering at Quinnipiac University. He completed a Ph.D. in Mathematics and another Ph.D. in Industrial and Systems Engineering at the University of Oklahoma. His pedagogical research interest includes technology and calculus education of STEM majors. He worked on several IRB approved pedagogical studies to observe undergraduate and graduate mathematics and engineering students' calculus and technology knowledge since 2011. His other research interests include nonlinear optimization, financial engineering, facility allocation problem, vehicle routing problem, solar energy systems, machine learning, system design, network analysis, inventory systems, and Riemannian geometry. 


\title{
Technology Choices of Undergraduate Engineering Students for Solving Calculus Questions
}

\author{
Emre Tokgöz \\ Emre.Tokgoz@quinnipiac.edu \\ Industrial Engineering, School of Engineering, Quinnipiac University, Hamden, CT, 06518
}

The use of technology is increasing day-by-day in educational environments and industry. Teaching theoretical concepts with computer program and calculator applications help students to understand the use of technology in various areas of engineering calculations. Different technologies can be used for solving different kinds of engineering problems. In this work, technology preferences of engineering undergraduate students who were enrolled at a mid-sized Northeastern U.S. institution for solving three different types of calculus problems are investigated with the emphasis on understanding technology education of these students' during their high school and university education. The qualitative results to be displayed in this research consist of students' written questionnaire and video recorded interview responses. The nature of quantitative results consist of probabilities that reflect the students' technology preferences and the variation analysis of the programming preferences across different research questions. The results presented in this paper help to determine and understand engineering students' technology choices for solving different calculus problems based on their technology education. The participants of this Institutional Review Board (IRB) approved research completed the third calculus course of a fourcourse calculus sequence. This article is a continuation of another IRB approved research that was conducted by the researcher at a large Midwest U.S. institution.

Key Words: Computer programming preference; Undergraduate education; Calculator; Matlab; Excel; TI calculators; Casio; Calculus

\section{Introduction}

Daily engineering challenges do not only require well-developed engineering practice but also require a good knowledge of mathematics and the use of right technology. Choosing the right technology to determine a solution to a problem matters from many perspectives including accuracy, practicality, and right approach to the solution. Many technologies such as Texas Instruments (TI) and Casio calculators; programming languages such as Matlab, Microsoft Excel, Java, and C++; web based resources such as Wolfram Alpha are widely used by students who are either required to use these technologies by their instructors. Calculators such as TI and Casio are widely used in the United States Universities or high schools (Tokgöz, 2012). It is crucial to know 
the mathematical theory to solve problems, however it is also important to know the appropriate technology to solve these problems. For instance, students can use Wolfram Alpha for graphing functions and do various calculations through a webpage (wolframalpha). Research on engineering students' technology preferences to solve mathematics problems is very limited on the contrary to the importance of the use of technology in engineering applications to solve mathematics problems. A research on STEM (Science-Technology-Engineering-Mathematics) majors' technology preferences to solve mathematics problems is conducted by Tokgöz (2015) Researchers such as Goosen (2004 and 2008), Ali (2007), Giangrande (2007), and Mannila (2006) focused on determining the best programming language to start teaching high school and undergraduate students. Clough (2002) and Maase \& High (2008) explored various technologies' education to engineers in undergraduate curriculums. Computer Science majors' mathematics problem solving skills using C programming language in the classroom is explored by Stockwell (2002). This article focuses on undergraduate and graduate students programming language preferences for solving mathematics problems. The only study similar to the one explained in this article is conducted by Tokgöz (2015) focusing on undergraduate and graduate STEM students technology preferences for solving calculus questions. The next section is devoted to detail the methodology followed for conducting the research, research objectives, the questions used for collecting the data on understanding participating students' technology preferences to solve calculus questions, and the main goals of the research.

\section{Research Methodology, Objectives \& Questions}

The IRB approved research explained in this article is conducted at a mid-sized North-Eastern university in the United States. Seventeen participants of this research majored in mechanical, industrial, or civil engineering. These participants completed the first three calculus courses of a four-sequence calculus. The research data was collected by an undergraduate student who was hired as a Research Assistant (RA) by the author/researcher during a spring school semester. A written questionnaire consisting of 14 questions was given by the RA to the participating students to be completed in about an hour. The author/researcher interviewed each participant approximately 30-40 minutes to further investigate the details of the written responses. Three out of seventeen participants did not participate in the video recorded interviews. The participating students are compensated for both their written and video recorded responses. The questionnaire consisted of calculus questions and technology preferences of the participants to solve calculus questions. In this article, all three questions related to the technology preferences of the participants will be analyzed qualitatively and quantitatively, the rest of the questionnaire questions will be analyzed in other articles. The main objectives of the three research questions analyzed in this article are the following: 
- Determining engineering majors' technology preferences for solving function graphing, definite integral, and mathematical series questions;

- Analysis of variation for participants' technology preferences when calculus concepts change. I.e. Do the students prefer a different technology (or not prefer at all) when calculus questions change?

- Identify participants' pool of technology knowledge and analyze how this knowledge base increased since high school. I.e. Research participants' progress of the technology knowledge from high school to university education.

The following three research questions are analyzed in this work that were also used in [Ref 10]. Q1) If you are required to draw the graph of a given function by using technology, what kind of technology would you use? Please either choose one of the following or write your own answer and explain why.
1. Calculator
6. Fortran
2. Excel
7. Matlab
3. $C$
8. LabVIEW
4. $C++$
9. Other
5. $C \#$

Q2) If there is a definite integral given, which one of the following would you prefer to use to calculate the given integral? Please circle the option and briefly explain why.
1. Computer Program (which program)
3. By hand
2. Calculator (which calculator)
4. Other (Please type)

If you are required to use a computer program to find the solution of the given definite integral, which computer language would you prefer to use?

Q3) If you needed to calculate numerical values of power series or error term graphs/values which method (algebraic calculations, computer program (please specify), calculator etc.) would you use? If you are required to pick a computer program what programming language would you prefer to use? Please either choose one of the following or write your own answer and explain why.
1. Calculator
6. Fortran
2. Excel
7. Matlab
3. $C$
8. LabVIEW
4. $\mathrm{C}++$
9. Other
5. $\mathrm{CH}$ 
The following are investigated for engineering educators and researchers to benefit from the data displayed in this work:

- Undergraduate engineering students' cognitive approach to technology preference;

- The technological shortcomings that arise in engineering education for solving mathematics problems;

- Changes in engineering students' technology preference from high school to university.

- The limitations of engineering students' technology knowledge.

The rest of the article is organized section-by-section for qualitative and quantitative analysis of participants' technology preferences to graph a function, solve definite integrals, and calculate power series or error terms. Students' written responses and transcribed video recorded interviews are displayed in these sections with the detailed analysis of variations in participants' technology preferences. The variation of technology preference with the change of the research question is also investigated. This analysis of variation is expected to show whether research participants' technology preference vary when questions change and their ability to use the right technology for the appropriate question.

\section{Technology \& Function Graphing}

Function graphing is one of the most fundamental concepts in engineering education. Beginners' calculus courses can be designed to cover function graphing by using only paper-pencil solution rather than using technology. Learning the right technology to solve mathematics problems is crucial for ease of solving engineering oriented mathematics problems in advanced engineering and mathematics courses as well as real-life applications. In this section, the responses of the seventeen participants' to the first research question will be analyzed qualitatively and quantitatively. The written and transcribed interview responses of the students will be displayed in this section for readers to have a better understanding of the collected data. The following table displays the responses of the participants during the written portion of the interviews.

\begin{tabular}{|c|c|l|}
\hline Participant \# & Choice & \multicolumn{1}{c|}{ Detailed explanation } \\
\hline 1 & Calculator & "Calculator (TI - 83+) or using grapher installed on all Macs" \\
\hline 2 & Matlab & $\begin{array}{l}\text { "I would choose Matlab for it is the easiest method to make } \\
\text { presentable graphs for reports..." }\end{array}$ \\
\hline 3 & Excel & $\begin{array}{l}\text { "Generally this software is available on all machines, where I have } \\
\text { only used Matlab at QU" }\end{array}$ \\
\hline 5 & $\begin{array}{c}\text { Calculator } \\
\text { (TI-84+) } \\
\& \text { Excel }\end{array}$ & $\begin{array}{l}\text { "It is what I am most comfortable with and consistently used it for } \\
\text { all classes" } \\
\text { hand. The calculator and Excel are both good basis for plotting data } \\
\text { and relatively easy to use." }\end{array}$ \\
\hline
\end{tabular}




\begin{tabular}{|c|c|c|}
\hline 6 & Calculator & $\begin{array}{l}\text { "I use a TI- } 84 \text { plus (I've had it for years but the new version ie even } \\
\text { more advanced!) I like being able to graph it, change the window, } \\
\text { and utilize the table..." }\end{array}$ \\
\hline 7 & Calculator & $\begin{array}{l}\text { "I have more experience in graphing and manipulating graphs. } \\
\text { With Excel and Matlab I have basic experience of plotting graphs } \\
\text { but not much analyzing these using basic functions." }\end{array}$ \\
\hline 8 & $\begin{array}{l}\text { Excel \& } \\
\text { Matlab }\end{array}$ & "Excel and Matlab I use the most in School." \\
\hline 9 & Calculator & $\begin{array}{l}\text { "I would use a TI- } 84 \text { plus because it is the calculator that I have } \\
\text { using for years and I know it well." }\end{array}$ \\
\hline 10 & Calculator & "TI 84 because that is what I am most comfortable with." \\
\hline 11 & Matlab & $\begin{array}{l}\text { "I am the most familiar with it and it provides more versatility then } \\
\text { Excel." }\end{array}$ \\
\hline 12 & Calculator & Casio $\mathrm{fx}-9750 \mathrm{GII}$ \\
\hline 13 & Matlab & $\begin{array}{l}\text { "It would normally be the calculator but the TI } 84 \text { isn't FE } \\
\text { approved so Matlab would be next, and once the data is entered } \\
\text { and graphed, it can easily be processed." }\end{array}$ \\
\hline 14 & Excel & $\begin{array}{l}\text { "Excel would be quicker for me to graph any given function than } \\
\text { a calculator." }\end{array}$ \\
\hline 16 & Matlab & "I have gotten good at that and it comes out neat and pretty." \\
\hline 17 & Calculator & $\begin{array}{l}\text { "TI } 84 \text { graphing calculator. I always have it with me and it is the } \\
\text { easiest thing. It is small and easily accessible..." }\end{array}$ \\
\hline
\end{tabular}

Participant 15 only circled Matlab and did not explain the response therefore this response is not displayed here.

Further investigation on participants' written responses during the video recorded interviews helped to understand how engineering majors choose technology and under what conditions they prefer to use the technology that they are aware of.

Interviewer: If you are required to draw the graph of a function by using Technology which one would you use? You are saying calculator...

Participant 1: Last semester during Calculus 3 I found this application on the Apple MacBook that is automatically installed; it is part of the utilities that you can type an equation on a 2D graph, 3D graph, just tons of different kinds and it made it very easy to draw them, move around, and find values. But otherwise the calculator TI 83+ would be fine.

Interviewer: If you are required to use a computer program what would you prefer?

Participant 1: I've been sort of messing around with Matlab, my friends at another school understand it but it's very confusing so if I could use a graphing calculator that would be great.

$\ldots$

Interviewer: For this question, you said you would use Matlab. 
Participant 2: Yes.

Interviewer: Did you learn Matlab before the university?...

Participant 2: No, I learned it here.

Interviewer: Do you consider using a calculator for this?

Participant 2: ... no because on Matlab I can get a more precise, or larger, range on my graphs...so I can see things a bit easier on larger scales which I think would have more observation data.

Interviewer: Did you learn any programming language when you were in high school?

Participant 2: I taught myself a bit of Python, but that was pretty much it.

Interviewer: ...If you're required to sketch the graph of a function, you're saying you would use Excel. And can you explain your answer overall with your explanation?

Participant 3: Yeah so the only time I've ever used Matlab is here at the University. So with work experience I normally use Excel...because they don't have Matlab.

Interviewer: Ok, I see. Would you rather use Matlab instead of Excel if it's available?

Participant 3: It would depend on the application. If it's a generic application I would use Excel. If I'm trying to do more advanced math I would use Matlab. And if I was doing statistics I have knowledge on a software called Minitab, which is a statistical software so I would use that.

...

Interviewer: ... Did you learn any type of technology when you were in high school? Did you use a calculator or any program language?

Participant 5: ...just the calculator.

Interviewer: Was it TI 84+?

Participant 5: Yes.

Interviewer: ... here you're saying calculator and/or Excel. Which one would you prefer to graph the function?

Participant 5: I would probably prefer a Calculator just because how I always did it in high school. I'm not as familiar with Excel.

...

Interviewer: ...If you are required to draw the graph of the function, you're saying you would use a calculator TI 84+. Would you use a programming language by any chance?

Participant 6: No. I've never learned a programming language, so I just said the calculator.

Interviewer: ... Did you use a calculator in high school?

Participant 6: Yeah.

Interviewer: ... Do you know any online program?

Participant 6: ... I had to use Logitech for a mathematics course, I think it was 300-the discrete math, so I had to kind of teach myself how to program using that. But that's not really graphing anything, it's a typesetting. ..

Interviewer: You said you would use Excel, Matlab, calculator and Labview. Which one would you prefer to use?

Participant 8: For graph. My calculator for sure.

Interviewer: And then?

Participant 8: Then I would probably use Excel, then Matlab and then Labview. Just because this is how I used stuff in the past. Labview I only touched briefly; Matlab I am getting a little better; Excel I am pretty good at and I used my calculator since $7^{\text {th }}$ grade... I used calculator and Excel when I was in high school; I haven't used and of Matlab or Labview in high school.

...

Interviewer: Do you know any programming languages?

Participant 10: I know Matlab and I used Labview a couple of times but I know Excel

Interviewer: Would you use anyone of those programs to solve the question? 
Participant 10: If I was going for speed I would use calculator but in terms of actually doing it right by spending time on it I would probably use Matlab because that is the one that I am best with among the others.

Interviewer: So then Matlab versus calculator, you would use calculator?

Participant 10: Yes

$\ldots$

Interviewer: Can you explain your answer please?

Participant 11: Yeah, I am most familiar with Matlab from what I learned and I would use that to draw the graph of the function because it gives me the most flexibility to deal with it because I can manipulate it in the way I want. The only other program that I am familiar with to do anything would be Excel. I just know that Matlab gives me more variability with what I want to do with the function.

Interviewer: Do you know how to use calculator?

Participant 11: Like a normal calculator, TI 84?

Interviewer: Yes. Which one would you prefer: Matlab or TI 84?

Participant 11: To just graph a function?

Interviewer: Yes.

Participant 11: For convenience just to draw the graph of the function I wouldn't necessarily pull out Matlab because I know I can get the job done with my calculator.

Interviewer: When did you learn using Matlab and calculator?

Participant 11: Calculator would be freshman year of high school and Matlab would be the freshman year of college. ...

Interviewer: You said you would use calculator Casio fx - 9750 GII. When did you learn using Casio?

Participant 12: When I came to America the first math course I took, the Professor asked for a graphing calculator and I went to a store and got one. For the whole school year I kept using Casio, that's why I would use it.

Interviewer: The same Casio?

Participant 12: No, the new technology.

Interviewer: When you want to sketch the graph of a function, would you use something else than that calculator?

Participant 12: No

$\ldots$

Interviewer: Can you please explain your answer here?

Participant 13: We have been using a lot of Matlab in my engineering program. When I was taking Calculus I was using a lot of TI 84 but that is not really a part of the FE exam that we are responsible for that we suppose to take soon. Just Matlab would be nice to graph this.

Interviewer: Which one is easier to use: calculator or Matlab?

Participant 13: Calculator

Interviewer: But you would still use Matlab?

Participant 13: Yeah, I guess that is where you are getting the real data.

...

Interviewer: You said you would use Excel to graph a function. Do you know how to use a calculator?

Participant 14: Yes.

Interviewer: You would rather use Excel instead of a calculator?

Participant 14: No, I would rather use a calculator. Excel would allow me to do more than what my calculator can do.

Interviewer: What type of calculator do you use?

Participant 14: TI 84.

Interviewer: What would be the list of your preference? 
Participant 14: I like the calculator but I feel like the calculator is limited. I wouldn't do calculator because I wouldn't know how many things to put in whereas in Excel after a certain point it is just beneficial to use Excel. It is a lot easier to put in numbers in Excel.

Interviewer: So you are talking about data oriented functions?

Participant 14: Yes, if it was a function of the form y equals to some function then I would use calculator.

Participants 7 and 9 preferred to use calculator for solving all three research questions. Participants 15-17 did not participate to the video recorded interviews, however their written responses clearly indicated their technology preferences. The next section is devoted to the qualitative and quantitative analysis of the collected data for determining technology preferences of the engineering students' function graphing.

\section{Analysis \& Summary of the Data}

Technology preferences of engineering undergraduate students' function graphing is influenced by their high school experiences. Approximately 59\% of the students preferred to use a TI graphing calculator while $65 \%$ of the participants preferred to use a calculator for function graphing. Approximately $29 \%$ of the participants preferred to use Matlab that they learned during their undergraduate education. The third, and the last, technology preference is Excel that was chosen by $12 \%$ of the participants. The transcribed data indicated participants' technology preferences depend on the complexity of the problem. Some of the participants' "just to sketch the graph of a function I wouldn't use Matlab" response indicated students' technology preferences to be driven by the complexity of the problem. Sketching the graph of a function appears as a challenging task for some of the students because of the coding aspect of Matlab. Some of the participants chose Matlab for its accuracy, strength, and convenience for obtaining proper graphing results. The written responses to this research question resulted in the following number of students and their technology preferences:

\begin{tabular}{|c|c|c|c|c|c|}
\hline Technology & Matlab & Excel & TI 83 + & TI 84+ & Calculator or Excel \\
\hline Number of users & 5 & 2 & 4 & 5 & 1 \\
\hline
\end{tabular}

National Instrument (NI) Labview was mentioned by several participants, however this choice was not listed as the first programming option to be considered for sketching the graph of a function. The interview results indicated several participants' preferences to choose a program that they learned to sketch a function just because they don't know any other programming language. Microsoft Excel was considered by several participants for function sketching with the justification that Excel can do more than what a graphing calculator can do. Majority of the participants preferred to use a calculator that they are familiar with from high school years instead of using programming languages such as Matlab, Excel, or N.I. Labview that they learned during their engineering undergraduate education. 
9. If there is a definite integral given, which one of the following would you prefer to use to calculate the given integral? Please circle the option and briefly explain why.

$$
\begin{aligned}
& \text { 1. Computer Program (which program) } \\
& \text { 2. Calculator (which calculator) } \\
& \text { 3. By hand } \\
& \text { 4. Other (Please type) } \\
& \text { If you are required to use a computer program to find the solution of the } \\
& \text { given definite integral, which computer language would you prefer to use? }
\end{aligned}
$$

$$
\text { matlab }
$$

\section{Response of Participant 8}

9. If there is \& definite integral given, which one of the following would you prefer to use to calculate the given integral? Please circle the option and brielly explain why.

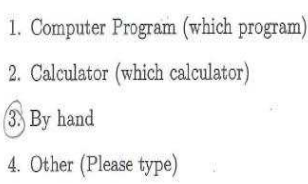
given definite integral, which computer language would you prefer to use?

$$
M A T L A B
$$

\section{Response of Participant 10}

9. If there is a definite integral given, which one of the following would you prefer to use to calculate the given integral? Please circle the option and briefy explain why.

1. Computer Program (which program)

2. Calculator (which calculator)

3. By hand

4. Other (Please type) put the probtum in google to see of the problent has been solvet

If you are required to use a computer program to find the solution of the given definite integral, which computer language would you prefer to use?

$$
\text { I have no idla }
$$

\section{Response of Participant 12}

9. If there is a definite integral given, which one of the following would you preer to use to calculate the given integral? Please circle the option and briefly explain why.

$$
\begin{aligned}
& \text { 1. Computer Program (which program) } \\
& \text { 2. Calculator (which calculator) } \\
& \text { 3. By hand Id Rather not use my hand, but that is the only way I have } \\
& \text { 4. Other (Please type) }
\end{aligned}
$$

If you are required to use a computer program to find the solution of the given definite integral, which computer language would you prefer to use?

$$
\text { Excel }
$$

\section{Response of Participant 14}

. If there is a definite integral given, which one of the following would you prefer to use to calculate the given integral? Please circle the option and briefly explain why.

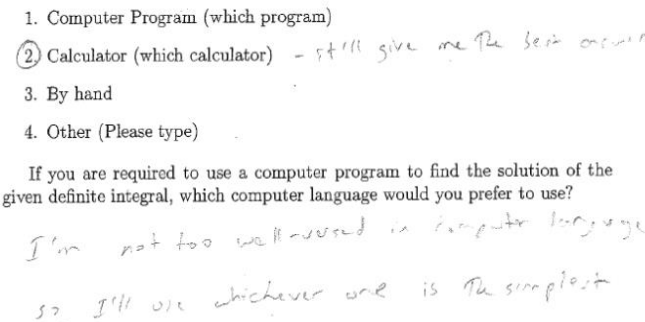

\section{Response of Participant 9}

9. If there is a definite integral given, which one of the following would you prefer to use to calculate the given integral? Please circle the option and briefly explain why.

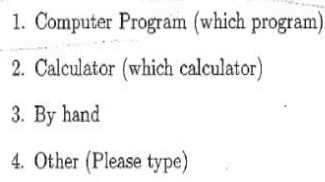

If you are required to use a computer program to find the solution of the given definite integral, which computer language would you prefer to use?

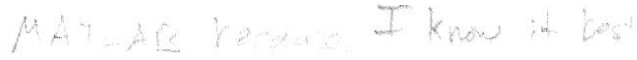

\section{Response of Participant 11}

9. If there is a definite integral given, which one of the following would you prefer to use to calculate the given integral? Please circle the option and briefy explain why.

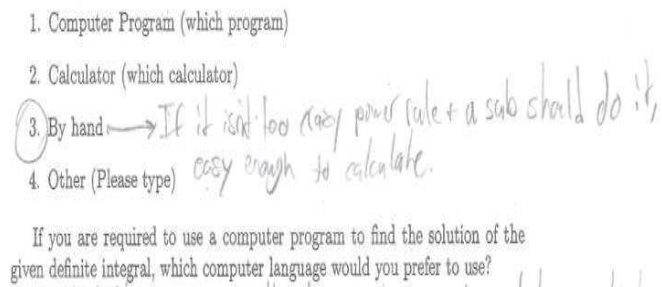

If you are required to use a computer program to find the solution of the given definite integral, which computer language would you prefer to use?

\section{Response of Participant 13}

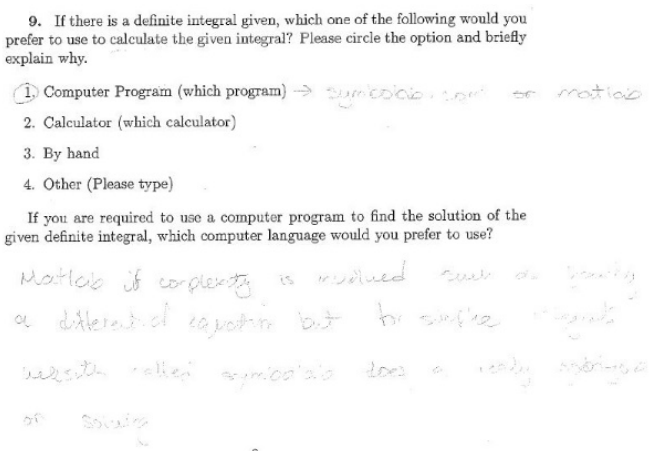

Response of Participant 15 
Participant 7 circled "by hand" option and wrote "calculator" as the computer programming preference. Participants 16 and 17 both chose to use Matlab and indicated its ease of use. The video recorded interviews helped to understand participants' technology preference reasons and the conditions under which they would prefer to use the corresponding technology.

Interviewer: If there's a definite integral given you're saying that you would solve it by hand?

Participant 1: Yes because normally I could use a calculator but there's a special function on TI 84 right now that you can plug in a definite integral so that looks exactly like an integral. So if it has the integral symbol and then you can plug in numbers right here and here and then your function which pretty much makes it a lot easier to read.

Interviewer: If you have a TI 83 then you're like hunting for random functions that you don't really understand the abbreviations for so it's easier just to do it by hand...A visual way of seeing the integral helps you and it is easier to actually program it or use a calculator whatever you are doing that helps you to visualize it better?

Participant 1: Yes

Interviewer: Okay. (Reads the response of the participant) "Java because it is what I know, possibly Matlab." Have you ever tried it with Java?

Participant 1: No I haven't, but that's what I've been studying in my engineering program for the past two years so I figured it might make it a bit easier.

..

Interviewer: You are saying you would use Wolfram Alpha for calculating the definite integral. Why Wolfram Alpha and not Matlab?

Participant 2: ...well I actually just learned integral integration using Matlab this week. We did it the easy way. But I've used Wolfram just as a source for checking my work for a long time, and it's really powerful with definite integrals. I can put in anything and it will tell me the answer. And it will also display a breakdown of the steps it took to get there, so if I'm stuck somewhere I can see what the next step is.

Interviewer: Does visual representation matter?

Participant 2: Sometimes. It depends on the specific integral or function.

Interviewer: Now, as you learned Matlab, would you rather choose Matlab or would you still go with Wolfram Alpha?

Participant 2: ...depends on what the application is. ...let's say it's something like a test, I obviously cannot use Wolfram Alpha, so I would use Matlab if I had that available. However, overall if I was to solve a complex problem... It kind of does that work for you...

Interviewer: ...if you decide to calculate a definite integral, you're saying that you would do it by hand. Can you explain your answer?

Participant 3: ... Ok...I suppose this one was black-and-white... If this was a simple integral that I would probably do it by hand. If it was complex that I would probably use Wolfram Alpha or Matlab or something.

Interviewer: ...Alright did you use any programming language in high school?

Participant 3: ...no.

Interviewer: Did you use a calculator in high school?

Participant 3: ... yes we used graphing calculators but I didn't have the knowledge of all of the functions that they did, so I would still do integrals by hand.

...

Interviewer: .... To be able to calculate definite integrals, you said you wouldn't use a computer program? Is that why you crossed it...

Participant 5: Yeah, I circled it and then I changed my mind. 
Interviewer: I see. You would use TI 84+ and you would use google to find a helpful website. Do you know any webpages?

Participant 5: Not off the top of my head, no.

Interviewer: ...Would you use Excel for this? Do you know how to use Excel?

Participant 5: No, that's why I wouldn't use it.

Interviewer: You are saying you would solve an integral question by hand or Matlab

Participant 8: Yeah, I just recently learned how to calculate integrals by using Matlab, it is much easier. I would rather do it that way because it is much easier... I don't mind doing integrals by hand as far as I remember the rules but I have to look them up sometimes

...

Interviewer: If you need to calculate a definite integral you are saying you would solve it by hand.

Participant 10: Yes, if I know how to solve it.

Interviewer: And when it comes to programming language you would use Matlab (based on the written response). Is there any other language that you would consider using?

Participant 10: Not really

...

Interviewer: Can you please explain your answer?

Participant 11: Looking at this know, I was thinking Matlab because I know how to do that but if it truly just to evaluate a definite integral I could easily do it by using a calculator...

...

Interviewer: In your response you said you have no idea and you chose the option "other." Can you please explain your answer?

Participant 12: The easiest way for me to see if the problem has whether a solution or not is to put it in Google.

Interviewer: How do you put the problem in google?

Participant 12: Try to use a key word

Interviewer: If you have a complicated integral problem how would you put it in Google?

Participant 12: There is a website called Wolfram Alpha that you can enter and calculate the integral.

Interviewer: Wouldn't you use calculator or solve it by hand?

Participant 12: No

Interviewer: Can you please explain your answer?

Participant 13: If it is kind of not too difficult to solve by hand. Putting in a calculator or program can kind of annoying. If you want a quick answer for a value then a calculator or a program is fine but if you want the actual equation to plug in different ranges later then calculating by hand is better.

Interviewer: Which one would you want to use: calculator or Matlab?

Participant 13: Probably calculator if you just want the value really quickly.

Interviewer: How do you decide to solve by using technology or by hand?

Participant 13: If you get into trigonometric functions then I would rather get into technology. Those can be pretty complicated.

...

Interviewer: Can you please explain your answer.

Participant 14: I would rather not use my hand but that is the only way that I know besides the other ways.

Interviewer: Do you know any programming languages?

Participant 14: I know Java but I never learned how to solve integrals using Java, I just learned basics of algebraic calculations.

Interviewer: You said Excel for the programming language. Do you know how to use Excel to solve integrals? 
Participant 14: No but if I were required I would choose Excel because I don't know Matlab and I don't like computer programming. Excel is simpler, it is easier for me.

The next section is devoted to the qualitative and quantitative analysis of the collected data for determining technology preferences of the engineering students' definite integral calculations.

\section{Analysis \& Summary of the Data}

A paper-pencil solution for determining definite integrals is preferred by $53 \%$ of the participants while $24 \%$ of the participants preferred to use Matlab and $12 \%$ of the participants chose to use calculator. The availability of Wolfram Alpha as one of the online free resources was mentioned by a couple of participants. The following table displays the technology and the corresponding number of users to solve a definite integral based on the written responses.

\begin{tabular}{|l|c|c|c|c|c|}
\hline Technology & Matlab & By hand & Calculator & Excel & Google \\
\hline Number of users & 4 & 9 & 2 & None & 1 \\
\hline
\end{tabular}

Some of the interview responses changed the table values above. For instance, Participant 8 stated "I just recently learned how to calculate integrals by using Matlab, it is much easier. I would rather do it that way because it is much easier." Some of the participants explained the conditions under which they would prefer to use technology: if the question appears to be simple then they would solve it by hand but if the question appears to be challenging then they would prefer to use technology. Such a preference depends on students' decision making and causes uncertainty in determining technology versus paper-pencil solution of the participants.

The next section is devoted to participating students' technology preferences for determining power series and error term results.

\section{Technology \& Power Series or Error Term Calculation Preferences}

Power series and error term calculations resulting from functions' power series expansions can be challenging. The technology that need to be used for such calculations may need to be more advanced than a simple calculator, therefore participants in this research are expected to have a better knowledge of qualified technology for calculating such numerical values. In this section, the written responses of the participants will be displayed first and the analysis of the written and interview data will be implemented next. The following table displays the responses of the participants during the written portion of the interviews. 


\begin{tabular}{|c|c|c|}
\hline Participant \# & Choice & Detailed explanation \\
\hline 1 & Calculator & "TI - 83+" \\
\hline 2 & Matlab & $\begin{array}{l}\text { "I would pick Matlab for it is the easiest and most comprehensive } \\
\text { when dealing with vector space and it would be the best at } \\
\text { calculating numerical series data." }\end{array}$ \\
\hline 4 & Calculator & "Calculator and algebraic calculations." \\
\hline 5 & $\begin{array}{l}\text { Calculator } \\
(\text { TI-84+) } \\
\text { \& Excel }\end{array}$ & $\begin{array}{l}\text { "Because I am not familiar with the other methods regarding this } \\
\text { function" }\end{array}$ \\
\hline 6 & Calculator & $\begin{array}{l}\text { "Calculator is easiest for me. I think doing it algebraically was very } \\
\text { difficulty... I am not sure about a computer program, I haven't had } \\
\text { to use any." }\end{array}$ \\
\hline 7 & $\begin{array}{l}\text { Other - } \\
\text { By hand }\end{array}$ & $\begin{array}{l}\text { "I would pick a calculator (for technology) because that is what I } \\
\text { am most familiar with." }\end{array}$ \\
\hline 8 & Excel & "I would most likely use Excel..." \\
\hline 9 & Calculator & "It is the one that I know the best." \\
\hline 10 & Calculator & "TI 84 because that is what I am most comfortable with." \\
\hline 11 & Matlab & "Because I learned this in Matlab." \\
\hline 13 & Matlab & $\begin{array}{l}\text { "I remember power series being being long and difficult, plug in } \\
\text { the equation to Matlab and let it do the work." }\end{array}$ \\
\hline 14 & Excel & $\begin{array}{l}\text { "I only know how to use Excel and... Excel allows me to solve } \\
\text { problems quicker." }\end{array}$ \\
\hline 17 & Matlab & "Most familiar with capabilities and functions" \\
\hline
\end{tabular}

Participant 3 did not have a written response to the question. Participants 10, 12, and 16 only circled calculator and wrote "TI 84", "Casio fx - 9750GII", and "TI 84+", respectively.

Participant 1:... You can graph it on the calculator and then you can find the integral by one of the trace methods. And I'll approximate that and then I'll try to do it by hand to see if I can get the same answer, so if I can find that then and if not then I'll say my reasoning behind why I think it'll approximate what I found with the graphing calculator.

Interviewer: Okay. Do you think the graphing calculator would give you the precise solution?

Participant 1: No but it would probably give me an approximate.

Interviewer: Ok. You prefer to use TI 83+ as before when you want to calculate power series or error term graphs and also you're saying you would use Java or Matlab as a program. Which one would you prefer to use in the setting? The calculator first or programming language first?...

Participant 1: Calculator.

Interviewer: Is there a simple function that helps you to calculate this?

Participant 1: ...I guess there is.

Interviewer: Okay. Did you ever use Java or Matlab?

Participant 1: No. 
Interviewer: This question is asking which one you would prefer to use if you calculate numerical values of power series or error terms and you are saying Matlab...

Participant 2: I am experienced in Matlab now but it's also because Matlab is really good at dealing with vector space and, well it's in a series and I can put that very easily into a matrix and do a lot of manipulation with it at that point.

...

Interviewer: If you needed to calculate numerical values of power series or error term graphs' values, you are saying you would use Matlab. Can you explain your answer here as well?

Participant 3: ... if I had to pick a computer program it would be Matlab because for power series I feel like those are more complex than something I would do in Excel and Matlab are the only two I have knowledge using...

Interviewer: Can you please explain your answer?

Participant 8: I would probably use Excel. I have never really needed to calculate particular numerical values or power series but in terms of error I have only calculated that one by using Excel or Matlab. I haven't done a lot of that earlier, I would say maybe once or twice besides kind of percent error but that is pretty straight forward, I can do that one by hand that is not what this question is asking.

Interviewer: So you would do it by hand?

Participant 8: If it is a simple error yeah but if it power series I have only done it once and it was with Matlab. $\ldots$

Interviewer: For calculating power series and error terms you said you would use calculator TI 84. Is that the one that you most comfortable with?

Participant 10: Yeah, I have been using it since high school

Interviewer: You wouldn't use Matlab?

Participant 10: No, it requires loops and all that and that is not really my favorite. I don't like hard coding...

$\ldots$

Interviewer: Would you use calculator for power series calculations?

Participant 11: Probably not, that would involve a lot of typing.

Interviewer: You said you would use the calculator that you mentioned before for number 5. Would you use anything else?

Participant 12: No, I don't know any of the others.

Interviewer: Do you know any other programming language?

Participant 12: Java

Interviewer: And you wouldn't use Java to calculate power series?

Participant 12: No

...

Participant 13: For something like power series you can loop it which can be helpful. There is a pattern in it. So you could assign a loop value and iterate through all of them. It would be a lot easier doing it this way I think. This is kind of how I would solve it.

Interviewer: ....Can you use calculator?

Participant 13: I wouldn't know how to do it on a calculator, no.

Interviewer: Is there any online resource that you would use.

Participant 13: There is Wolfram Alpha that I can use.

...

Participant 14: I would just use Excel; same as the first two questions. It would be quicker to use Excel if you have numbers. Even though calculator would be always my first choice if it was a quick solve.

Interviewer: Do you know how to use calculator for power series calculations?

Participant 14: No, I do not know. 
Some of the participants' transcribed responses are not displayed due to the similarity of their responses to the first two research questions covered. Qualitative and quantitative analysis of the collected data for determining technology preferences of the engineering students' power series and error term related calculations will be covered in the next.

\section{Analysis \& Summary of the Data}

Numerical calculations regarding to the power series expansion of functions or error terms related to power series of functions can require advanced knowledge of programming use. The participants were expected to choose a programming language and justify their preference of the programming language to solve power series questions. The following table displays the type of technology that participants prefer to use and the corresponding number of users.

\begin{tabular}{|l|c|c|c|c|c|}
\hline Technology & Matlab & By hand & Calculator & Excel & No response \\
\hline Number of users & 5 & 1 & 8 & 2 & 1 \\
\hline
\end{tabular}

Calculator is preferred by $50 \%$ of the participants while $31.3 \%$ of the students chose to use Matlab and $12.5 \%$ decided to use Excel for determining a numerical solution for a power series or error term calculation problem. The analysis of the interviews indicated some of the students' lack of experience with power series calculations, therefore these participants chose the technology that they are most familiar with. Some of the participants hesitated to use a computer programming language due to the need of writing a code to solve the problem.

\section{Variation Analysis of Participants' Preferences}

The number of Matlab users is uniform across all three research questions Q1-Q3 with a small variation. The maximum variation appeared on the calculator and paper-pencil solution preferences. Hardly any variation in the number of users (10 and 8 users) is observed for calculator preference to solve Q1 and Q3, however the variation was high for Q2 (2 users). There was no variation in the number of users (2 users) for using Excel to solve Q1 and Q3 but a high variation is observed for using Excel on Q2 (no users). Similarly, hardly any variation in the number of users is observed for the paper-pencil solution preference to solve Q1 and Q3 but a high variation is observed for Q2 (9 users). The following table summarizes the number of users' technology preferences to solve Q1 - Q3 based on Calculator, Matlab, Excel, and paper-pencil categories. 


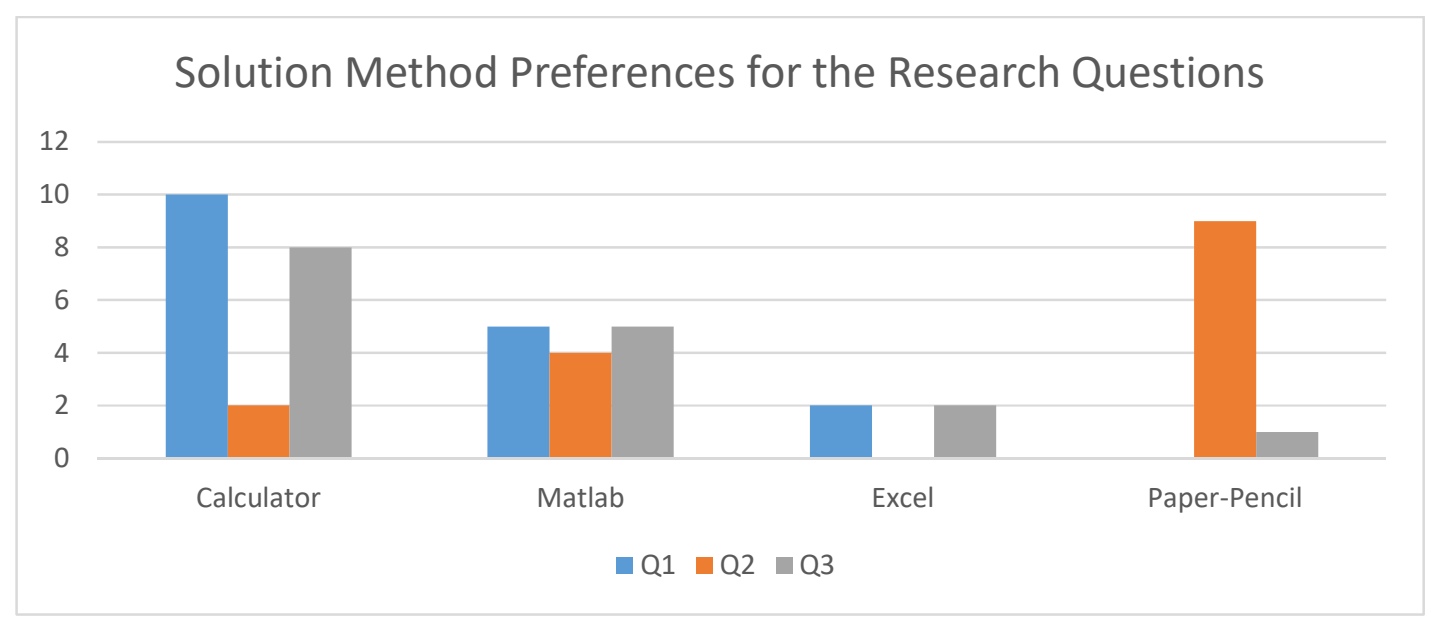

\section{Conclusion \& Future Work}

In this work, 17 engineering undergraduate students' technology preferences to solve various calculus questions are investigated at a mid-sized North-Eastern university in the United States during a Spring school semester similar to the work of Tokgöz (2015). Institutional Review Board's approval was required to conduct the research because the research subjects are students. All 17 participants written responses to a written questionnaire consisting of 14 questions was collected by an undergraduate Research Assistant (RA) who was hired by the researcher. Among the participants, 14 out of 17 agreed to be interviewed by the researcher for about 30-40 minutes to further investigate the details of their written responses. The researcher asked participants to explain their responses during the interviews and asked additional questions as needed. The main objectives of the three research questions analyzed in this article were to observe the following:

- Engineering majors technology preferences for solving function graphing, definite integral, and mathematical series related questions;

- The variation of participants' technology preferences when the calculus concept changes. I.e. Do the students prefer different technologies (or not prefer at all) when the calculus questions change?

- Determine participants' list of technology knowledge and analyze how this knowledge base increased in time. I.e. The increasing progress of the technology knowledge of the participants from high school to university.

Engineering educators and researchers are expected to benefit from the data displayed in this work in several different ways: 
- An understanding of undergraduate engineering students' cognitive technology preferences for solving calculus questions;

- Recognize the shortcomings that arise in engineering education for solving mathematics problems by using technology;

- Progress in engineering students' technology knowledge from high school to university.

- Recognize the limitations of engineering students' technology knowledge and improve their knowledge accordingly.

Participants' research questionnaire consisted of 11 calculus and 3 technology preference questions. The three technology preference questions from the questionnaire and the corresponding participant preferences based on the written responses are listed below:

Q1) If you are required to draw the graph of a given function by using technology, what kind of technology would you use?

\begin{tabular}{|l|c|c|c|c|c|}
\hline Technology & Matlab & Excel & TI $83+$ & TI 84+ & Calculator or Excel \\
\hline Number of users & 5 & 2 & 4 & 5 & 1 \\
\hline
\end{tabular}

Q2) If there is a definite integral given, which one of the following would you prefer to use to calculate the given integral?

\begin{tabular}{|l|c|c|c|c|c|}
\hline Technology & Matlab & By hand & Calculator & Excel & Google \\
\hline Number of users & 4 & 9 & 2 & None & 1 \\
\hline
\end{tabular}

Q3) If you needed to calculate numerical values of power series or error term graphs/values which method (algebraic calculations, computer program (please specify), calculator etc.) would you use? If you are required to pick a computer program what programming language would you prefer to use?

\begin{tabular}{|l|c|c|c|c|c|}
\hline Technology & Matlab & By hand & Calculator & Excel & No response \\
\hline Number of users & 5 & 1 & 8 & 2 & 1 \\
\hline
\end{tabular}

Some of the technologies and online resources that students mentioned during the interviews included Matlab, TI, Excel, Wolfram Alpha, Casio, Java, and Labview similar to the work of Tokgöz (2015). Among these options, the participants listed Matlab, TI calculators, Excel, Wolfram Alpha, and a Casio calculator to solve the three research questions. The interview response analysis to all three questions indicated the following outcomes: 
- Some of the participants chose the technology that they are most familiar with even if they never used that technology for solving the corresponding calculus problem;

- Technology versus paper-pencil solution is a decision making process and depends on the type of question: in the case when the question is "simple" participants" preferred paperpencil solution and technology otherwise.

- TI calculators were the most popular option for graphing functions, and power series and error term calculations.

- Paper-pencil solution was the most popular option for calculating definite integrals.

- Matlab appeared to be the best second option for solving all three questions. The hesitation of several participants' Matlab preference was due to its' programming aspect.

In conclusion, it is important to increase technology knowledge base of engineering students for them to be able to choose the right technology to solve different problems. For instance, Wolfram Alpha is an online free resource that all students can use as long as they know its availability and how to use it. Further investigation similar to this research is needed to have a good understanding of students' technology preferences. A hybrid approach that includes technology and instructor appears to be eminent for a better educational system in the future.

\section{References}

Ali, A.I. and Kohun, F. "Suggested Topics for an IS Introductory Course in Java.” In: Proceedings of the Informing Science and Information Technology Education Joint Conference (2005), 33-49, 2007.

Clough, David E., “ChE's Teaching Introductory Computing to ChE Students - A Modern Computing Course with Emphasis on Problem Solving and Programming," Proceedings of the ASEE Annual Conference \& Exposition, 2002.

Giangrande, E.: CS1 programming language options. Journal of Computing Sciences in Colleges, 22, 153-160, 2007.

Goosen, L. "Criteria and guidelines for the selection and implementation of a first programming language in high schools," PhD thesis, North-West University (Potchefstroom Campus), 2004.

Goosen, L. "A brief History of Choosing First Programming Languages," In IFIP International Federation for Information Processing,; History of Computing and Education 3; Editor J. Impagliazzo; (Boston: Springer), 269, 167-170, 2008.

Maase, Eric L. and High, Karen A. "Active Problem Solving and Applied Research Methods in a Graduate Course on Numerical Methods," 42(1) 23-32, 2008. 
Mannila, L. and de Raadt, M. "An objective comparison of languages for teaching introductory programming," In: Proceedings of the 6th Baltic Sea conference on computing education research. 3237. ACM Press, New York, 2006.

Stockwell, W. F. "The effects of learning "C" Programming on College Students' Mathematical Skill," Ph.D. dissertation, University of Oklahoma, Norman, OK, 2002.

Wolframalpha, Online available resource @ https://www.wolframalpha.com/

Tokgöz, E. 'Undergraduate and Graduate STEM Majors' Technology Preference for Solving Calculus Related Questions,” ASEE Annual Meeting Proceedings, Seattle, Washington, Paper I.D. \#: 12668, 2015. 